\section{El reto de las transferencias de colgajos lïbres en pacientes quemados ¿Cuál es el mejor momento para la cirugía?}

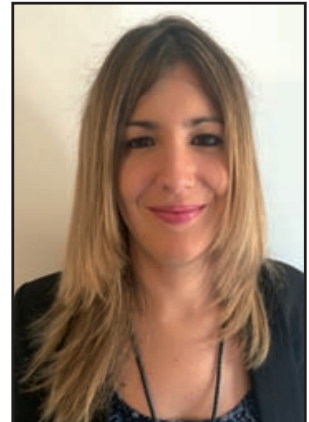

Villaverde Doménech, M.E.

\author{
Villaverde-Doménech, M.E.*, Simón-Sanz, E.**, Delgado-Ruíz, T.*, \\ Pérez-Ramos, L.***, Safont-Albert, J.****
}

\section{Resumen}

La transferencia de colgajos libres es una herramienta fundamental dentro del arsenal reconstructivo para el tratamiento quirúrgico del gran quemado. Durante años ha existido controversia sobre la influencia del momento de la cirugía sobre la tasa de complicaciones, así como el alto riesgo de complicaciones al realizar reconstrucciones con colgajos libres en pacientes quemados.

Diseñamos un estudio retrospectivo de las reconstrucciones quirúrgicas mediante colgajos libres realizadas en nuestra Unidad de Quemados del Hospital Universitari y Politecnic La Fe, de Valencia (España) en el periodo comprendido entre 2001 y 2013, recogiendo todos los datos relativos al paciente, a la lesión, al procedimiento reconstructivo, a los resultados y relacionando todo ello con el momento en el cual se realizó la cirugía.

Para el análisis de los datos y asociaciones entre distintas variables usamos tablas de contingencia y aplicamos el test de independencia de Chi-cuadrado.

Exponemos además 2 casos clínicos representativos de la cirugía reconstructiva practicada en pacientes quemados

Recogimos en total datos de 21 procedimientos de reconstrucción mediante colgajo libre en pacientes quemados. En ninguno se realizó reconstrucción primaria inmediata (en los primeros 5 días), mientras que la reconstrucción primaria temprana (entre el día 5 y el 21 ) se realizó en el $28,57 \%$ de los casos, la primaria intermedia (entre el día 21 y las 6 semanas) en el 28,57\%, la secundaria (más de 6 semanas) en el 9,5\%, y la cirugía de secuelas en el $33,33 \%$ de los casos. Las tasas de complicaciones mayores y menores fueron respectivamente $19,05 \%$ y $9,5 \%$. El $50 \%$ de complicaciones mayores ocurrieron en el periodo de reconstrucción primaria temprana, mientras que no registramos ninguna en el periodo primario intermedio. El resto de complicaciones se repartió a partes iguales entre el periodo secundario y la cirugía de secuelas (ambas con el 25\%).

No encontramos asociación estadísticamente significativa entre las variables en ninguna de las comparaciones realizadas

En conclusión, creemos que la reconstrucción mediante colgajos libres en pacientes quemados presenta un riesgo más elevado de complicaciones mayores cuando se realiza en el periodo primario temprano (entre los días 5 y 21 tras la quemadura).

\section{Palabras clave Colgajos libres, Transferencia de colgajos vascularizados, Quemaduras, Complicaciones en quemaduras.}

Nivel de evidencia científica

$3 b$

* Médico Interno Residente.

** Jefe de Sección.

*** Licenciada en Estadística. Máster en Bioestadística.

**** Jefe del Servicio.

Servicio de Cirugía Plástica y Reconstructiva. Hospital Universitari y Politécnic La Fe Valencia España.

Trabajo galardonado con el premio en la $1^{\text {a }}$ Convocatoria del Reconocimiento al Tratamiento Quirúrgico del Gran Quemado convocado por la Revista Cirugía Plástica Ibero-Latinoamericana bajo el patrocinio de Mediwound ${ }^{\circledR}$ y con los auspicios de la Fundación Docente SECPRE, entregado en $50^{\circ}$ Congreso de la Sociedad Española de Cirugía Plástica, Reparadora y Estética (SECPRE) celebrado en Toledo (España) del 22 a 25 de mayo del 2015.
Free flap transfer is an essential tool within the reconstructive repertoire for the surgical treatment of major burn patients. For a long time there has been controversy about the influence of the timing over the complication rate, as well as the increased complication risk when we perform reconstruction by means of free flaps in burn patients.

We designed a retrospective study about free flap reconstructions perforcia (Spain), in the period from 2001 to 2013, collecting data related to the patient, the lesion, the reconstructive procedure, the results, and all this data related to timing.

For the data analysis and associations between the different variables we used contingency tables and applied the Chi-square independence test.

In addition we present 2 representative clinical reports about burn reconstructive surgery.

Data from 21 reconstructive procedures with free flaps in burn patients were collected.

Immediate primary reconstruction (in the first 5 days) is not performed in any patient, while early primary reconstruction (from day 5 to 21 ) was performed in $28,57 \%$ of cases; the intermediate primary reconstruction (from day 21 to 6 th week) was performed in $28.57 \%$, the secondary reconstruction (more than 6 weeks) in $9,5 \%$ and sequelae surgery in 33,33\% of the cases. Major and minor complication rate were $19,05 \%$ and $9,5 \%$ respectively; $50 \%$ of major complications happened in early primary reconstruction period, whereas no complication is registered in intermediate primary period, the rest of complications are equally divided between secondary reconstruction period and sequelae surgery (both of them $25 \%$ ).

No statistically significant association between the variables is found in any of the comparisons performed.

In conclusion, we think that reconstruction with free flaps applied to burn patients presents a higher major complication rate when performed in the early primary period (from day 5 to day 21 after the burn). med in our Burns Unit at the Hospital Universitari y Politecnic La Fe, Valen-

$$
\begin{aligned}
& \text { Free tissue flap, Vascularised flap } \\
& \text { transfer, Burn surgery, } \\
& \text { Complications in burned patients. }
\end{aligned}
$$




\section{Introducción}

Las primeras publicaciones sobre la utilización de colgajos libres en pacientes quemados datan de 1975 $(1,2)$. En un principio se utilizaron casi exclusivamente para el tratamiento de secuelas, pero con el tiempo se integraron como una técnica más en el tratamiento del quemado agudo.

En la cirugía reconstructiva de los quemados la transferencia de colgajos vascularizados, a pesar de no ser una técnica de uso cotidiano, constituye una herramienta muy útil para el cirujano plástico. La versatilidad, variabilidad de los colgajos libres y su adaptabilidad a cada lesión en concreto, han incrementado en los últimos años sus indicaciones en la cirugía del quemado $(3,4)$. Los colgajos libres en general tienen una clara indicación como cobertura de tejidos nobles (hueso, articulaciones, tendones, nervios y estructuras vasculares), que muchas veces quedan expuestos tras desbridamientos agresivos. En estos casos es frecuente que no dispongamos de colgajos pediculados locorregionales, y la cobertura con injertos de piel parcial estaría abocada al fracaso. Además, la transferencia de colgajos vascularizados en cirugías de salvamento de miembros ha evitado numerosas amputaciones en pacientes quemados, así como acortamientos en muñones con exposición ósea y déficit de tejidos blandos. Las características particulares de la lesión o defecto van a determinar la elección del tipo de colgajo libre (tamaño del defecto, tipo de tejido, coloración de la piel, etc.) (5).

$\mathrm{Al}$ revisar la literatura al respecto, observamos que durante años ha existido controversia sobre la utilización de colgajos libres en la cirugía reconstructiva en pacientes quemados dada la alta incidencia de complicaciones registradas. Además, en numerosas publicaciones vemos que la tasa de complicaciones varía dependiendo del momento elegido para la reconstrucción. Los estudios coinciden en que la mayor incidencia de complicaciones se registra en el periodo comprendido entre el $5^{\circ}$ y $21^{\circ}$ día tras la quemadura (4-6). Otros autores sin embargo, afirman que si la técnica es adecuada (algo que se presupone de entrada), el lecho está correctamente desbridado y hay un buen control de la infección y de la inflamación, no hay variaciones sobre el riesgo de fracaso de los colgajos al aplicarlos en pacientes quemados (7).

Al margen de esta controversia debemos centrarnos en el paciente quemado, que por lo general es un enfermo grave y muchas veces se encuentra en situación crítica, por lo que aún aceptando que la tasa de pérdida de colgajos libres fuera más elevada en este tipo de pacientes, tal y como apoyan diversos autores (8), con frecuencia el colgajo libre va a ser nuestra arma más definitiva para evitar una amputación u otras complicaciones graves, por lo que el riesgo/beneficio estaría justificado en muchos casos (exponerse a un teórico riesgo aumentado de pérdida o complicaciones del colgajo a cambio de evitar complicaciones aún más graves). En este punto, son las quemaduras eléctricas de alto voltaje las que más morbilidad causan al afectar estructuras profundas (tendones, nervios, vasos sanguíneos, huesos o articulaciones), provocando importantes secuelas funcionales e incluso amputaciones si no se realiza una cobertura temprana con tejido bien vascularizado (9-11). De hecho, de entre las quemaduras, las eléctricas de alto voltaje son las que presentan mayor riesgo de amputación (12). La reconstrucción en un solo tiempo mediante transferencia de colgajos vascularizados permite el cierre precoz de las heridas, la movilización temprana de las articulaciones y el inicio de la rehabilitación, obteniendo así mejores resultados en cuanto a la recuperación funcional de los pacientes, hospitalizaciones más cortas y disminuyendo la tasa de amputaciones.

Nuestro objetivo con el presente estudio es hacer una revisión retrospectiva de las reconstrucciones mediante transferencia de colgajos vascularizados libres realizadas en la Unidad de Quemados del Hospital Universitari y Politecnic La Fe, de Valencia (España) entre agosto de 2001 y agosto de 2013, recogiendo una serie de datos relativos al paciente, a la lesión y al tratamiento practicado. Calcularemos la tasa de complicaciones de los colgajos libres en este grupo de pacientes quemados y analizaremos si existe alguna relación entre las complicaciones sufridas y el momento en el que se realizó la reconstrucción.

Además exponemos 2 casos clínicos representativos de 2 modalidades de reconstrucción. El primero, una reconstrucción de tipo funcional en el periodo primario intermedio y el segundo, una cirugía de secuelas de tipo estético.

\section{Material y método}

Llevamos a cabo un estudio retrospectivo de casos de pacientes quemados ingresados en nuestra Unidad de Quemados que precisaron reconstrucción con colgajo libre vascularizado en el periodo comprendido entre agosto de 2001 y agosto de 2013 para evaluar las características demográficas de los pacientes, las causas de la quemadura y las características de la lesión, las indicaciones de cirugía, los tipos de colgajos utilizados, el momento en que se realizó la reconstrucción, y los resultados de la reconstrucción, incluyendo las complicaciones. El estudio se llevó a cabo en la Unidad de Quemados del Hospital Universitari y Politecnic La Fe de Valencia (España), que es unidad de referencia del Sistema Nacional de Salud acreditada por el Ministerio de Sanidad desde 2008 y cuenta con 46 años de experiencia en el tratamiento de quemados, desde que fuera fundada en 1969. La tasa media de asistencia especializada de esta unidad entre los años 2012 y 2014 ha sido de 1707 urgencias/ año, con una tasa media de ingresos de 133 pacientes/año.

La información recogida procedió tanto de historias clínicas en papel como en registro electrónico; la revisión de los historiales clínicos se realizó siguiendo los 
protocolos de nuestra institución y cumpliendo con la Ley de Protección de Datos. Incluimos todos los pacientes mayores de 18 años a los cuales se les había realizado un colgajo libre durante el periodo de estudio, considerando cada procedimiento reconstructivo de modo independiente (en algunos pacientes se había realizado más de un colgajo libre). Registramos también una serie de datos como: edad, sexo, superficie corporal quemada, localización y características de la zona receptora o lesionada, indicación del colgajo, tipo de colgajo utilizado, momento en el que se realiza la cirugía, tiempo de estancia hospitalaria y complicaciones (infección, necrosis total y/o parcial, y alteraciones vasculares).

Analizamos las complicaciones en función del momento elegido para realizar la reconstrucción con la finalidad de ver si existía alguna relación entre estos dos factores. Consideramos varios periodos en la reconstrucción de una quemadura aguda: reconstrucción primaria cuando se realiza en las 6 semanas posteriores al traumatismo y que a su vez puede ser: inmediata si se realiza en los 5 primeros días, temprana si se realiza entre 5-21 días, intermedia si se realiza más allá de 21 días, y reconstrucción secundaria, que es toda reconstrucción realizada más allá de 6 semanas. Por otro lado estaría la cirugía de las secuelas de quemaduras.

Para el análisis de los datos y relaciones entre distintas variables usamos tablas de contingencia, y como mé- todo estadístico aplicamos test de independencia de Chicuadrado de Pearson $\left(\chi^{2}\right)$.

\section{RESULTADOS}

Recogimos un total de 21 procedimientos de reconstrucción mediante colgajo libre realizados en 19 pacientes quemados. La media de edad de dichos pacientes fue de 47,24 +/- 6,18 años (intervalo de confianza, I.C. al $95 \%$ ), con un rango entre los 18 y los 80 años; 14 varones $(66,6 \%)$ y 7 mujeres $(33,3 \%)$.

Según la etiología, la causa más frecuente de las quemaduras fue; la eléctrica de alto voltaje con 8 casos (38\%), seguida de la llama en 5 casos $(23,8 \%)$, la explosión en 4 casos (19\%), aceite en 2 casos $(9,5 \%)$ y química en 2 casos (9,5\%) (Gráfica 1, Tabla I). La extensión media de las quemaduras fue del $20 \%+/-7,27 \%$ de la superficie corporal total (I.C. al 95\%), con un rango de valores entre 2 y $55 \%$ de superficie corporal quemada.

En cuanto a la localización de la zona a reconstruir, la más frecuente fue en miembros con 14 casos $(66,6 \%)$. Observando los subgrupos hubo 8 reconstrucciones de miembros superiores $(38,1 \%)$ frente a 6 de miembros inferiores $(28,57 \%)$; 3 reconstrucciones craneofaciales $(14,29 \%)$ y 3 reconstrucciones axilares $(14,29 \%)$; por último, 1 caso de reconstrucción mamaria $(4,76 \%)$ (Gráfica 1, Tabla I).

Gráfica 1.

Resumen de las características de las lesiones y de los procedimientos realizados en pacientes quemados que precisaron reconstrucción mediante transferencia de colgajo vascularizado.

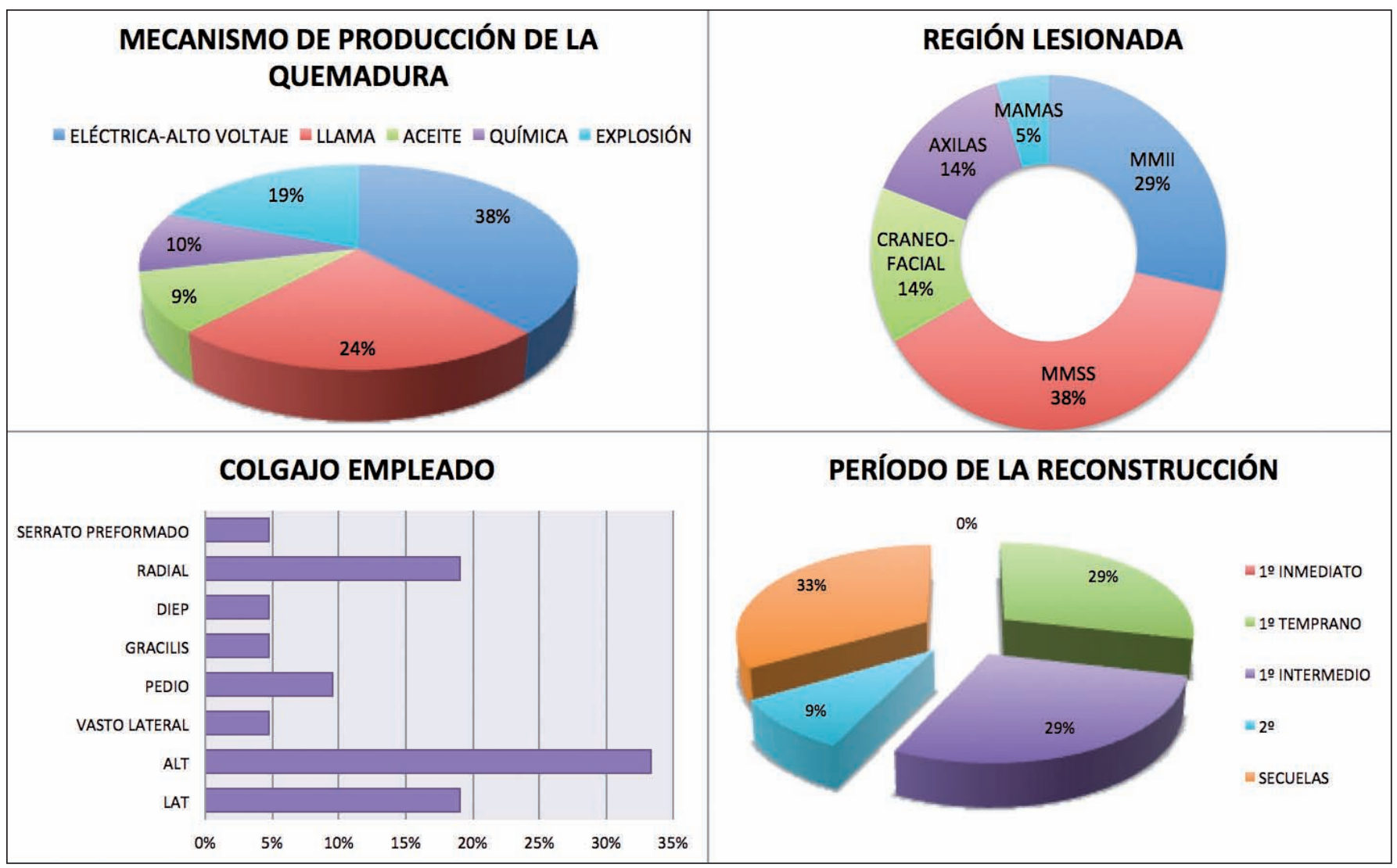


Tabla I. Demografía y características de la lesión

\begin{tabular}{|c|c|c|}
\hline EDAD (años) & \multicolumn{2}{|c|}{$47,24+/-6,18$ años (IC=95\%) } \\
\hline \multirow{2}{*}{ Sexo } & Mujeres & $7(33,3 \%)$ \\
\hline & Hombres & $14(66,6 \%)$ \\
\hline \multirow{5}{*}{ Etiología de la quemadura } & Alto voltaje & $8(38 \%)$ \\
\hline & Llama & $5(23,8 \%)$ \\
\hline & Explosión & $4(19 \%)$ \\
\hline & Aceite & $2(9,5 \%)$ \\
\hline & Química & $2(9,5 \%)$ \\
\hline $\begin{array}{l}\text { Extensión de la quemadura } \\
\text { (SCT) }\end{array}$ & \multicolumn{2}{|c|}{$20+/-7,27 \%(\mathrm{IC}=95 \%)$} \\
\hline \multirow{6}{*}{ Localización de la lesión } & $\begin{array}{c}\text { Miembros } \\
\text { (superiores e } \\
\text { inferiores): }\end{array}$ & $14(66,6 \%)$ \\
\hline & $\begin{array}{l}\text { Miembros } \\
\text { superiores }\end{array}$ & $8(38,1 \%)$ \\
\hline & $\begin{array}{l}\text { Miembros } \\
\text { inferiores }\end{array}$ & $6(28,57 \%)$ \\
\hline & Craneofacial & $3(14,29 \%)$ \\
\hline & Axilas & $3(14,29 \%)$ \\
\hline & Mamas & $1(4,76 \%)$ \\
\hline
\end{tabular}

Tabla II. Indicaciones, momento de la cirugía y complicaciones

\begin{tabular}{|c|c|}
\hline \multicolumn{2}{|c|}{ INDICACIÓN DE CIRUGÍA } \\
\hline Exposición de tejidos nobles & $14(66,6 \%)$ \\
\hline Corrección de bridas retráctiles & $4(19 \%)$ \\
\hline Reconstrucción facial & $2(9,5 \%)$ \\
\hline Reconstrucción mamaria & $1(4,75 \%)$ \\
\hline \multicolumn{2}{|c|}{ TIPOS DE COLGAJO } \\
\hline ALT & $7(33,3 \%)$ \\
\hline LAT & $4(19 \%)$ \\
\hline RADIAL & $4(19 \%)$ \\
\hline PEDIO & $2(9,5 \%)$ \\
\hline OTROS & $4(19 \%)$ \\
\hline \multicolumn{2}{|c|}{ MOMENTO DE LA CIRUGÍA } \\
\hline $1^{\circ}$ Inmediato & 0 \\
\hline $1^{\circ}$ temprano & $6(28,57 \%)$ \\
\hline $1^{\circ}$ intermedio & $6(28,57 \%)$ \\
\hline $2^{\circ}$ & $2(9,5 \%)$ \\
\hline Secuelas & $7(33,3 \%)$ \\
\hline \multicolumn{2}{|c|}{ COMPLICACIONES } \\
\hline Pérdida total del colgajo & $4(19,05 \%)$ \\
\hline $\begin{array}{l}\text { Complicaciones menores } \\
\text { (necrosis parcial, congestión } \\
\text { venosa) }\end{array}$ & $2(9,5 \%)$ \\
\hline \multicolumn{2}{|c|}{ ESTANCIA HOSPITALARIA (DİAS) } \\
\hline $54,24+/-16,02(\mathrm{IC}$ & \\
\hline
\end{tabular}

Respecto a las indicaciones de esta cirugía, en un 66,6\% (14 casos) su fin era la cobertura de estructuras nobles: óseas, tendinosas, o nerviosas; en un 19\% (4 casos) corrección de bridas retráctiles; en un 9,5\%
( 2 casos) reconstrucción facial; y en un 4,76\% ( 1 caso) reconstrucción mamaria. Dentro del grupo de cobertura de estructuras nobles, 7 casos precisaron solo cobertura de tejido óseo expuesto (33,3\%); 3 casos cobertura de tejido óseo y tendinoso (14,28\%); 2 exclusivamente de tendones $(9,5 \%)$; y por último otros 2 de tendón y nervio $(9,5 \%)$.

Los colgajos libres más utilizados fueron el anterolateral de muslo (ALT) en 7 casos $(33,3 \%)$, el de lattisimus dorsi (LAT) en 4 casos (19\%), el radial en 4 casos (19\%), el pedio o dorsal del pie en 2 casos $(9,5 \%)$, y otros varios que se usaron en una proporción menor: serrato preformado, DIEP, gracilis, y vasto lateral, cada uno de ellos en 1 caso, lo que supone el 4,75\% del total (Gráfica 1, Tabla II).

Por lo que se refiere al momento en que se realizó la cirugía, en ninguno de los pacientes se practicó reconstrucción primaria inmediata (es decir, en los 5 primeros días), siendo de preferencia la reconstrucción primaria, ya fuera temprana: 6 casos $(28,57 \%)$ o en el periodo intermedio: 6 casos $(28,57 \%)$. Se realizaron reconstrucciones secundarias (más allá de las 6 semanas) en 2 casos $(9,5 \%)$ y cirugía de las secuelas en 7 casos $(33,3 \%)$ (Gráfica 1, Tabla II).

La estancia media hospitalaria registrada fue de 54,24 días +/- 16,02 (I.C. al 95\%), siendo los valores máximo y mínimo 7 y 132 días respectivamente. La tasa de complicaciones mayores (necrosis de colgajo total) fue de $19,05 \%$ (4 casos); todos los casos complicados se debieron a complicaciones vasculares: 2 casos por trombosis arterial precoz (en las primeras 48 horas), y 2 casos por insuficiencia venosa con congestión y fenómenos trombóticos tardíos (de 48 horas a 7 días tras la cirugía), que finalmente provocaron la pérdida total del colgajo. En 2 de las reconstrucciones $(9,5 \%)$ hubo complicaciones menores que se resolvieron. Uno de los casos se trató de una necrosis parcial de los bordes del colgajo que se resolvió con desbridamiento marginal y curas seriadas; en el otro caso se produjo congestión venosa temporal que se resolvió mediante incisiones de drenaje y aplicación de heparina de bajo peso molecular (20 UI/12 horas durante los 3 primeros días y $20 \mathrm{UI} / 24$ horas del $4^{\circ}$ al $5^{\circ}$ día).

Dentro del análisis de las complicaciones mayores y del momento en el que se realizó la cirugía, el 50\% de complicaciones mayores ( 2 casos) ocurrieron en reconstrucciones realizadas en el periodo primario temprano, mientras que en el periodo de reconstrucción primaria intermedia no ocurrió ninguna. El resto de complicaciones mayores se repartieron a partes iguales entre las realizadas en el periodo secundario ( 1 caso, $25 \%$ ) y en la cirugía de secuelas (1 caso, 25\%) (Tablas II y III).

Estudiamos los distintos datos y relaciones entre variables usando tablas de contingencia y aplicando el test de Chi-cuadrado de Pearson, utilizando la simulación de Montecarlo para obtener el p-valor (dada la baja frecuencia observada y el elevado número de categorías de las variables). 
Tabla III. Resumen de los datos recogidos

\begin{tabular}{|c|c|c|c|c|c|c|c|c|c|c|}
\hline № & EDAD & SEXO & $\begin{array}{l}\text { MECANISMO DE } \\
\text { QUEMADURA }\end{array}$ & $\begin{array}{c}\text { TBSA } \\
\text { QUEMADA }\end{array}$ & ZONA RECEPTORA DE COLGAO & INDICACIÓN COLGAJO & TPO COLGAO & TIMING: & $\begin{array}{l}\text { ESTANCIA HOSPITALARIA } \\
\text { (DIAS) }\end{array}$ & $\begin{array}{l}\text { COMPLICACIONES } \\
\text { COLGAJO }\end{array}$ \\
\hline 1 & 46 & VARÓN & $\begin{array}{l}\text { ELÉCTRICA-ALTO } \\
\text { VOLTAEE }\end{array}$ & $4 \%$ & PIE IZQUIERDO (MIII) & $\begin{array}{l}\text { COBERTURA ÓSEA } \\
\text { (METATARSIANOS) }\end{array}$ & ALT & INTERMEDIO & 44 & No \\
\hline 2 & 45 & VARÓN & LLAMA & $16 \%$ & PIERNA IZQ (MIII) & $\begin{array}{l}\text { COBERTURA ÓSEAY } \\
\text { TENDINOSA }\end{array}$ & VASTO LATERAL & INTERMEDIO & 105 & No \\
\hline 3 & 64 & MUJER & ACEITE & $12 \%$ & $\begin{array}{l}\text { UNIDAD ESTÉECA: MEIILAA } \\
\text { DERECHA }\end{array}$ & RECONSTRUCCIÓN FACIAL & RADIAL & SECUELAS & 29 & No \\
\hline 4 & 65 & MUJER & ACEITE & $12 \%$ & $\begin{array}{l}\text { CANTOS INTERNOS, DORSO NASAL, } \\
\text { PÁRPADOS SUPERIORES }\end{array}$ & RECONSTRUCCIÓN FACIAL & PEDIO & SECUELAS & 18 & NO \\
\hline 5 & 32 & MUJER & EXPLOSIÓN & $55 \%$ & PIERNA IZQUIERDA (MII) & $\begin{array}{c}\text { COBERTURA ÓSEA (TIBIA) } \\
\text { YARTICULAR }\end{array}$ & LAT & TEMPRANO & 132 & NECROSIS COLGAJO \\
\hline 6 & 32 & MUJER & EXPLOSIÓN & $55 \%$ & PIERNA IZQUIERDA (MIII) & $\begin{array}{c}\text { COBERTURA ÓSEA (TIBIA) } \\
\text { YARTICULAR }\end{array}$ & GRACILIS & TEMPRANO & 132 & No \\
\hline 7 & 48 & VARÓN & $\begin{array}{l}\text { ELÉCTRICA-ALTO } \\
\text { VOLTAEE }\end{array}$ & $8 \%$ & $\begin{array}{l}\text { REGIÓN FRONTO-PARIETO } \\
\text { TEMPORAL DERECHA }\end{array}$ & COBERTURA ÓSEA-CALOTA & LAT & INTERMEDIO & 50 & NO \\
\hline 8 & 49 & VARÓN & $\begin{array}{l}\text { ELÉCTRICA-ALTO } \\
\text { VOLTAJE } \\
\end{array}$ & $7 \%$ & $\begin{array}{c}\text { ANTEBRAZO DERECHO, CARA VOLAR } \\
\text { (MSD) }\end{array}$ & $\begin{array}{c}\text { COBERTURA TENDINOSAY } \\
\text { N.MEDIANO } \\
\end{array}$ & RADIAL & TEMPRANO & 50 & No \\
\hline 9 & 32 & VARÓN & EXPLOSIÓN & $20 \%$ & CUELLO Y AXILA DERECHA & \begin{tabular}{|c|} 
CORRECCIÓN DE BRIDA \\
RETRÁCTIL
\end{tabular} & ALT & SECUELAS & 7 & No \\
\hline 10 & 18 & VARÓN & $\begin{array}{l}\text { ELÉCTRICO-ALTO } \\
\text { VOLTAEE }\end{array}$ & $20 \%$ & $\begin{array}{l}\text { REGIÓN DISTAL DE ANTEBRAZO } \\
\text { IZQUIERDO (MSI) }\end{array}$ & $\begin{array}{c}\text { COBERTURA TENDINOSAY } \\
\text { N.MEDIANO }\end{array}$ & RADIAL & SECUNDARIO & 61 & NO \\
\hline 11 & 32 & VARÓN & $\begin{array}{l}\text { ELÉCTRICA-ALTO } \\
\text { VOLTAEE }\end{array}$ & $35 \%$ & CODO DERECHO (MSD) & COBERTURA OSEA & LAT & INTERMEDIO & 75 & No \\
\hline 12 & 44 & VARÓN & $\begin{array}{l}\text { ELÉCTRICA-ALTO } \\
\text { VOLTAEE }\end{array}$ & $5 \%$ & $\begin{array}{l}\text { MUNÓN DE AMPUTACIÓN DE 3ERY } \\
\text { 40 DEDO PIE DERECHO (MID). } \\
\end{array}$ & COBERTURA ÓSEA & RADIAL & TEMPRANO & 38 & No \\
\hline 13 & 58 & MUJER & LLAMA & $12 \%$ & AXILA DERECHA & \begin{tabular}{|c|} 
CORRECCIÓN DE BRIDA \\
RETRÁCTIL \\
\end{tabular} & ALT & SECUELAS & 24 & No \\
\hline 14 & 42 & MUJER & LLAMA & $50 \%$ & MAMA IZQUIERDA & RECONSTRUCCIÓN MAMA & DIEP & SECUELAS & 38 & No \\
\hline 15 & 52 & VARÓN & LAMA & $21 \%$ & $\begin{array}{c}\text { REGIÓN CUBITAL ANTEBRAZO } \\
\text { DERECHO (MSD) }\end{array}$ & $\begin{array}{l}\text { EXPOSICIÓN ÓSEAY } \\
\text { TENOINOSA }\end{array}$ & ALT & INTERMEDIO & 100 & NECROSIS PARCIAL \\
\hline 16 & 58 & VARÓN & LLAMA & $10 \%$ & AXILA IZQUIERDA & $\begin{array}{l}\text { CORRECCIÓN DE BRIDA } \\
\text { RETRÁCTIL }\end{array}$ & ALT & SECUELAS & 12 & NECROSIS COLGAJO \\
\hline 17 & 38 & VARÓN & $\begin{array}{l}\text { ELÉCTRICA-ALTO } \\
\text { VOLTAJE }\end{array}$ & $7 \%$ & $\begin{array}{l}\text { DORSO DE PIE Y ANTEPIÉ DERECHO } \\
\text { (MID) }\end{array}$ & $\begin{array}{l}\text { EXPOSICIÓN ÓSEAY } \\
\text { TENDINOSA }\end{array}$ & ALT & INTERMEDIO & 79 & No \\
\hline 18 & 40 & VARÓN & $\begin{array}{l}\text { ELÉCTRICA-ALTO } \\
\text { VOLTAEE }\end{array}$ & $20 \%$ & ANTEBRAZO DERECHO (MSD) & EXPOSICIÓN TENDINOSA & ALT & TEMPRANO & 62 & NECROSIS COLGAJO \\
\hline 19 & 55 & VARÓN & EXPLOSIÓN & $41 \%$ & $\begin{array}{c}\text { BRIDAS MF E IF DE MANO DERECHA } \\
\text { MSD } \\
\end{array}$ & \begin{tabular}{|c|} 
CORRECCIÓN DE BRIDA \\
RETRÁCTIL \\
\end{tabular} & \begin{tabular}{|c|} 
SERRATO PREFORMADO \\
CON EXPANSOR \\
\end{tabular} & SECUELAS & 28 & CONGESTIÓN VENOSA \\
\hline 20 & 62 & MUJER & QUIMICA & $2 \%$ & DORSO DE MANO DERECHA (MSD) & $\begin{array}{c}\text { EXPOSICIÓN TENDINOSA } \\
\text { (EXTENSORES) }\end{array}$ & PEDIO & SECUNDARIO & 12 & NECROSIS COLGAJO \\
\hline 20 & 80 & VARÓN & QUÍMICA & $7 \%$ & $\begin{array}{l}\text { MUNOÓN AMPUTACIÓN MANO } \\
\text { IZQUIERDA (MSI) }\end{array}$ & $\begin{array}{l}\text { EXPOSICIÓN ÓSEAY } \\
\text { ARTICULAR }\end{array}$ & LAT & TEMPRANO & 43 & NO \\
\hline
\end{tabular}

Comparamos la variable tasa de complicaciones mayores (necrosis total, infección, alteraciones vasculares) con las siguientes variables para ver si existía asociación con alguna de ellas: con el mecanismo de quemadura, con las regiones lesionadas, con el tipo de colgajo utilizado y con el momento de la reconstrucción. Los resultados fueron respectivamente: Chi cuadrado $\left(\chi^{2}\right)=2,0305$, $\mathrm{p}$-value $(\mathrm{p}-\mathrm{v})=0,9055 ;=1,5441, \mathrm{p}-\mathrm{v}=0,9295 ;=3,6287$, $\mathrm{p}-\mathrm{v}=0,911 \mathrm{y}=3,5515, \mathrm{p}-\mathrm{v}=0,3913$, no encontrando asociación estadísticamente significativa entre las variables en ninguna de las comparaciones.

Para completar el trabajo presentamos 2 casos clínicos que ilustran la complejidad del tratamiento quirúrgico reconstructivo de los pacientes quemados, reconstrucciones que incluyen múltiples cirugías y suponen un gran reto clínico para una Unidad de Quemados. Se trata de 2 modalidades de reconstrucción: una de tipo funcional para cobertura de calota expuesta que se llevó a cabo en el periodo primario intermedio, y una cirugía de tipo estético de secuelas faciales.

Caso 1. Varón de 48 años con quemadura eléctrica de alta tensión en el contexto de un accidente laboral: transformador eléctrico que entra en contacto con la región parieto-temporal derecha del paciente produciéndole una quemadura de tercer grado en la zona de unos $11 \mathrm{~cm}^{2} \mathrm{de}$ superficie. Presenta hueso craneal expuesto y quemado (Fig. 1), así como quemaduras de tercer grado en hombro, superficie anterior del tórax y miembro inferior derecho, con marca de salida de corriente en la rodilla.

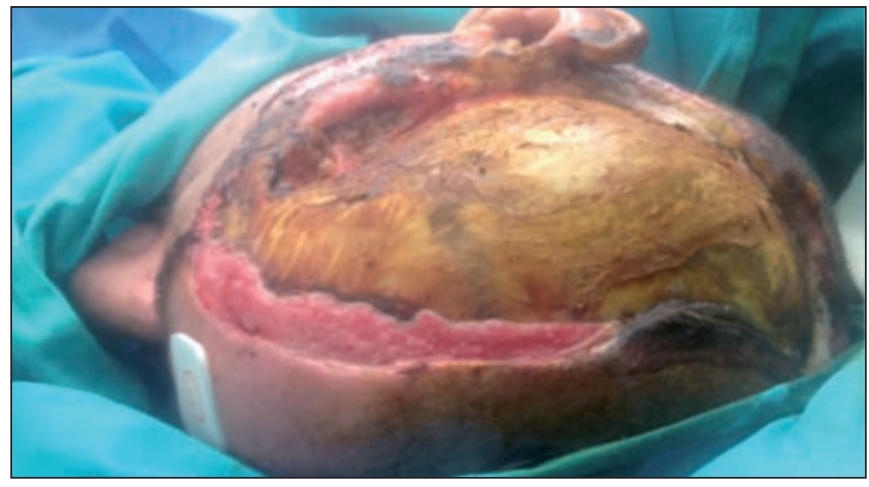

Fig. 1. Quemadura eléctrica de tercer grado en región fronto-parietotemporal con hueso craneal expuesto y quemado.

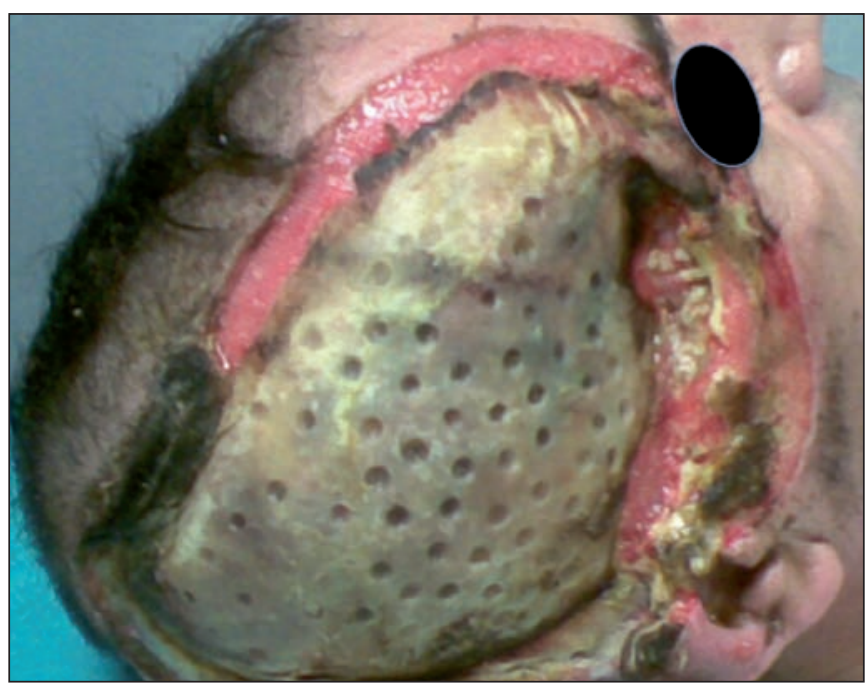

Fig. 2. Calota de región fronto-parieto-temporal expuesta tras desbridamiento de quemadura. Se realizaron fresados hasta médula del diploe. 
Tras la estabilización del paciente comenzó el proceso reconstructivo con curas seriadas y 2 intervenciones quirúrgicas para desbridamiento y cobertura de las quemaduras corporales con injertos de piel parcial. Estas 2 intervenciones se realizaron el $6^{\circ}$ y el $20^{\circ}$ día tras la quemadura. Como consecuencia del desbridamiento quedó hueso fronto-parieto-temporal expuesto de dudosa

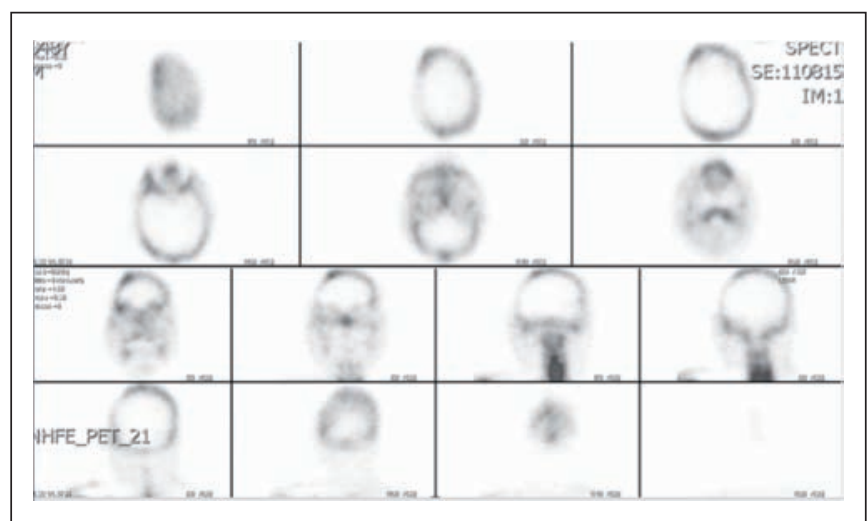

Fig. 3. SPECT TAC tras administración de $20 \mathrm{mCi}$ de 99mTc-HDP IV, con captación disminuida en el área fronto-parietal derecha, indicativa de viabilidad ósea ( $3^{\mathrm{a}}$ semana). viabilidad, realizándose fresados de su superficie hasta la medular (Fig. 2). En la tercera semana de ingreso se realizó SPECT TAC de cráneo tras administración intravenosa de $20 \mathrm{mCi}$ de $99 \mathrm{mTc}-\mathrm{HDP}$, identificando en la región parieto-occipital derecha captación disminuida respecto a la región contralateral, indicativa de viabilidad ósea de la zona (13) (Fig. 3). Esta prueba es muy útil en pacientes con quemaduras eléctricas tan profundas para determinar la viabilidad o no del hueso afectado. En cualquier caso, en base a nuestra experiencia en quemaduras eléctricas que afecten a hueso craneal, y tal y como también apoyan algunos estudios (14), la actitud que debemos tomar es conservadora con el desbridamiento, independientemente de los resultados del SPECT TAC, ya que se ha observado regeneración ósea en quemaduras de calota con tratamiento conservador y una cobertura adecuada.

El día 30 tras el traumatismo se realizó cobertura con colgajo libre de lattisimus dorsi con isla de piel anastomosado a la arteria y vena faciales y con injertos de piel sobre el músculo restante. El paciente permaneció ingre-

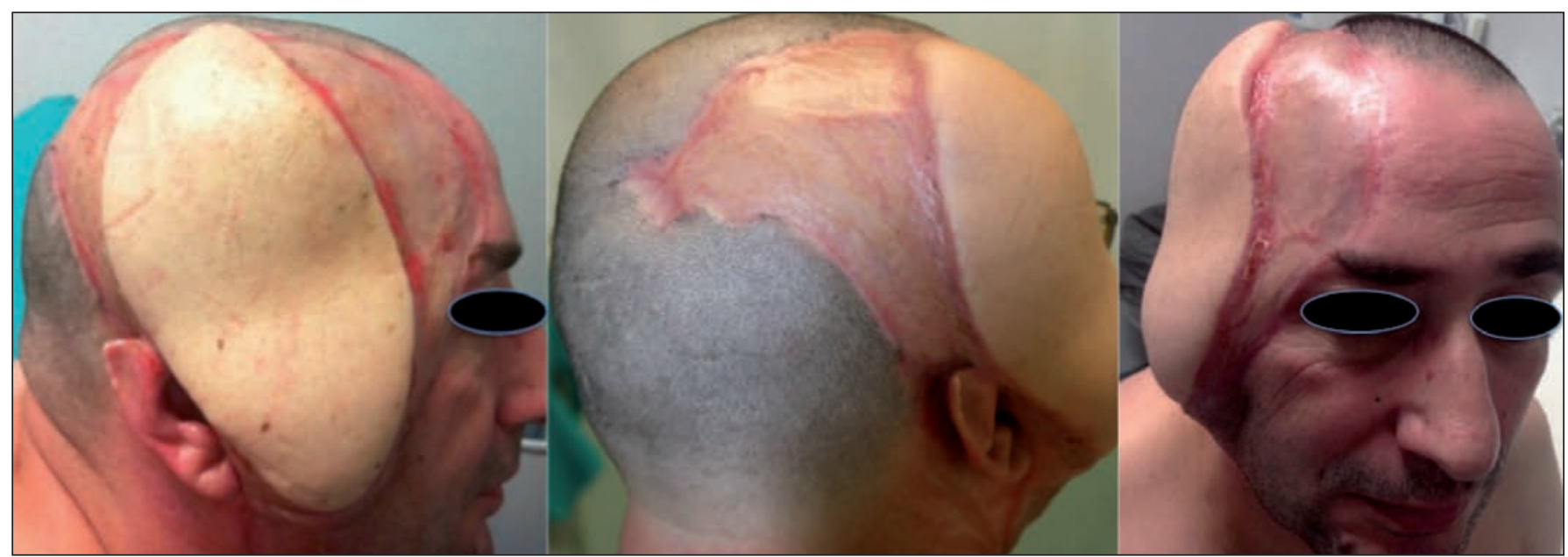

Fig. 4. Cobertura de defecto con colgajo libre de lattisimus dorsi, con isla de piel e injertos de piel parcial.
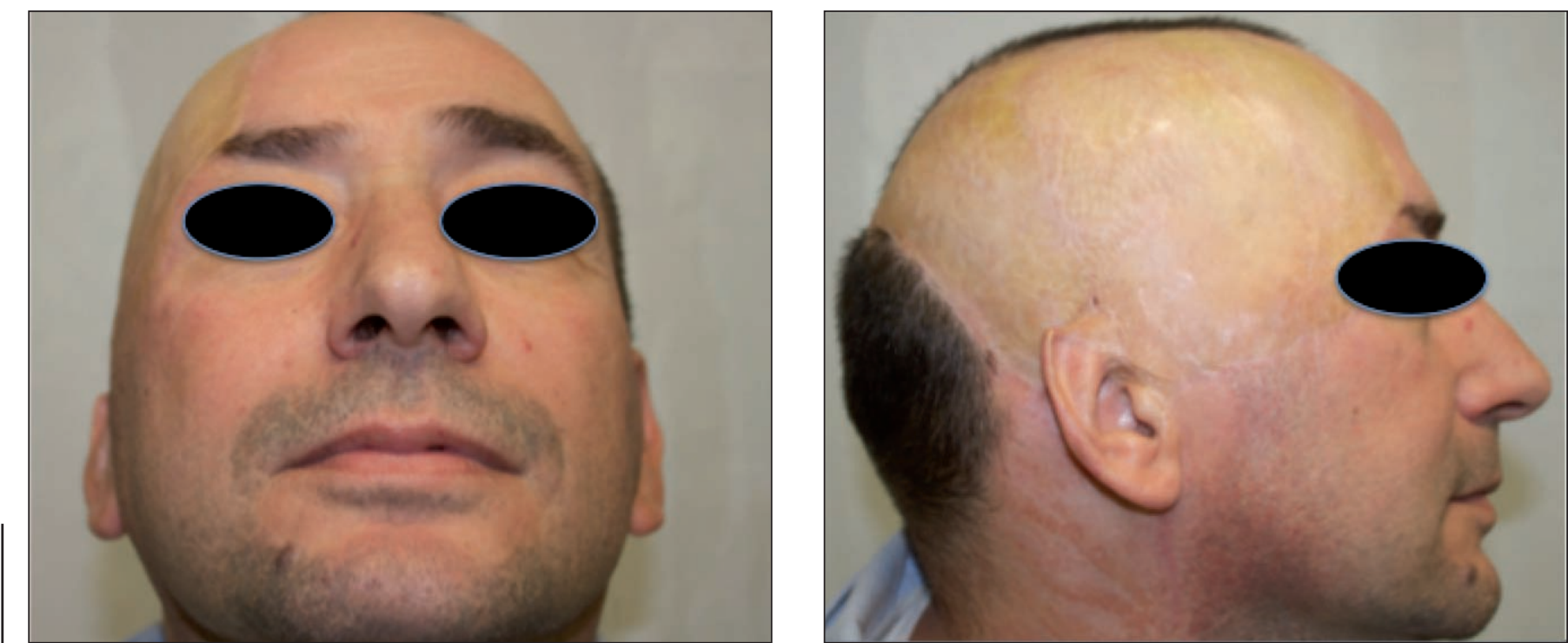

Fig. 5. Aspecto final a los 26 de la quemadura inicial, tras cirugías de remodelación de colgajo, incluyendo adelgazamiento, lipectomía e injertos autólogos de tejido adiposo 
sado en la Unidad de Quemados 3 semanas más, y dada su buena evolución y su adecuada recuperación fue dado de alta hospitalaria (Fig. 4).

En los meses posteriores se realizaron cirugías de refinamiento: en el $8^{\circ}$ mes tras la quemadura se practicó remodelación del colgajo de lattisimus dorsi mediante adelgazamiento y escisión de grasa, así como un colgajo de trasposición en isla cutánea para reconstrucción del hélix derecho; en el mes 18 se realizó un injerto autólogo de tejido adiposo para tratar las secuelas cicatriciales secundarias a la quemadura (depresiones suprarrotuliana, en muslo y antebrazo), así como para mejorar el aspecto del colgajo injertando el tejido adiposo bajo el colgajo (región fronto-parietal derecha); por último en el mes 25 se realizó un nuevo lipoinjerto bajo el colgajo, con lo que mejoró mucho el resultado de la reconstrucción en cuanto a volumen, textura, apariencia del colgajo y de la región parieto-temporal (Fig. 5), con un alto grado de satisfacción por parte del paciente y de los cirujanos. El resultado final se obtuvo a los 26 meses de la quemadura inicial.

Caso 2. Mujer de 64 años con importantes secuelas por quemaduras en cara sufridas 2 meses antes durante un ataque epiléptico mientras cocinaba y caerse en aceite hirviendo. Presenta grandes cicatrices hipertróficas y ectropión bilateral que no respondió al tratamiento inicial (3 intervenciones de desbridamiento e injerto los días 8,12 y 18 tras la quemadura): mala respuesta y recidiva del ectropión.

En primer lugar, 2 meses y medio tras la quemadura, se realizó resección de cicatrices hipertróficas y de bridas faciales (Fig. 6) y reconstrucción de la unidad estética de la mejilla derecha mediante colgajo libre radial, siendo los vasos receptores la arteria facial y la vena temporal superficial (Fig. 7). A los 5 meses de la quemadura se realizó una nueva intervención en la cual se extirparon las bridas y contracturas de la glabela y de los párpados superiores, y se reconstruyeron ambos párpados superiores e inferiores y glabela con injertos de piel total. En el $9^{\circ}$ mes tras la quemadura se realizó el siguiente tiempo quirúrgico: exéresis de bridas y cicatrices remanentes en cantos internos, dorso nasal y párpados, y cobertura de la superficie cruenta con un colgajo microquirúrgico de dorso de pie de un tamaño inicial de $10 \times 5,5 \mathrm{~cm}$, diseñado a medida para cubrir totalmente la lesión, y obteniendo muy buena cobertura gracias a la adaptabilidad del colgajo, su delgadez y pliabilidad (Fig. 8). Siete días antes se había preparado un asa vascular de arteria radial y vena basílica procedente del colgajo radial previo, a la cual se realizó transferencia vascularizada del colgajo mediante anastomosis T-T; para mejorar el drenaje venoso se suturó la vena safena del colgajo pedio a la yugular externa.

El resultado final, tanto en el postoperatorio inmediato a los 3 meses (Fig. 9) como a largo plazo 2 años después (Fig. 10), fue satisfactorio ya que se consiguió estabilizar las cicatrices y evitar la progresión de las bridas y la cicatrización hipertrófica.
Es importante recordar la complejidad de la reconstrucción facial, y la gran importancia que tiene en este sentido respetar las unidades estéticas de reconstrucción (15).

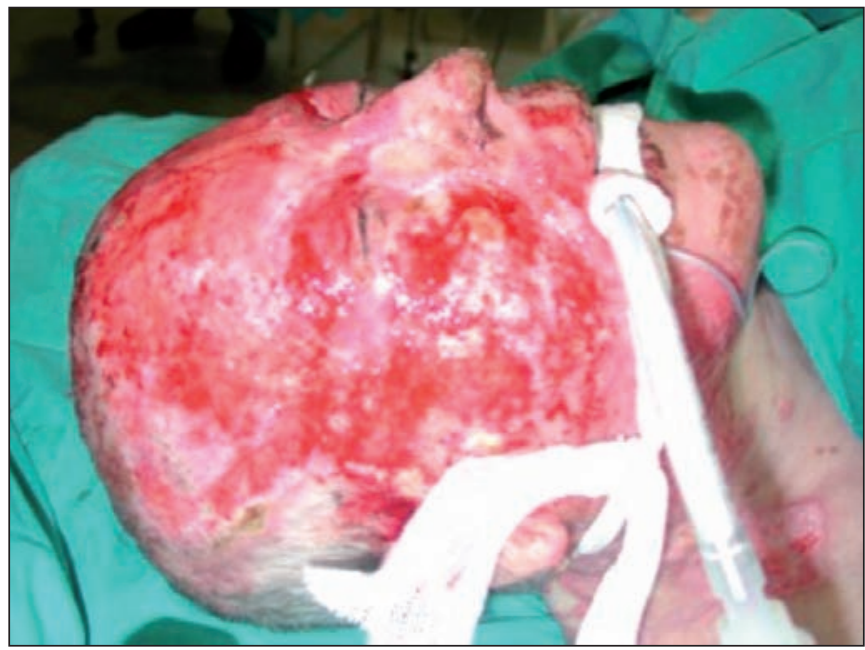

Fig. 6. Resección de bridas cicatriciales y cicatrices hipertróficas faciales.

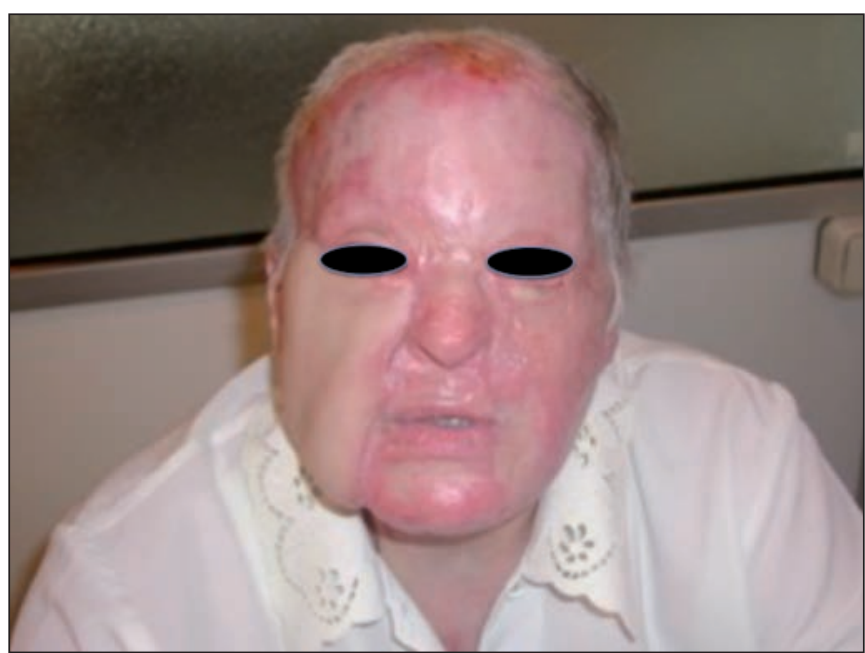

Fig. 7. Reconstrucción de mejilla derecha mediante colgajo radial libre.

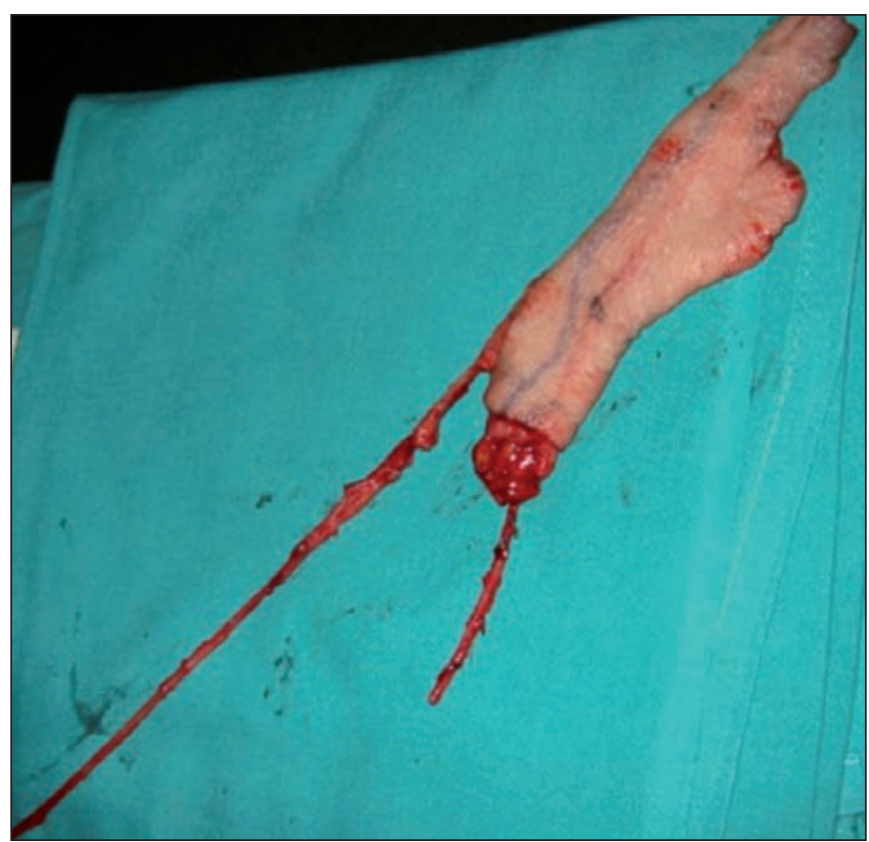

Fig. 8. Colgajo libre de dorso de pie. Obsérvese diseño, pliabilidad y delgadez que le permiten su adaptabilidad al lecho de la lesión facial. 


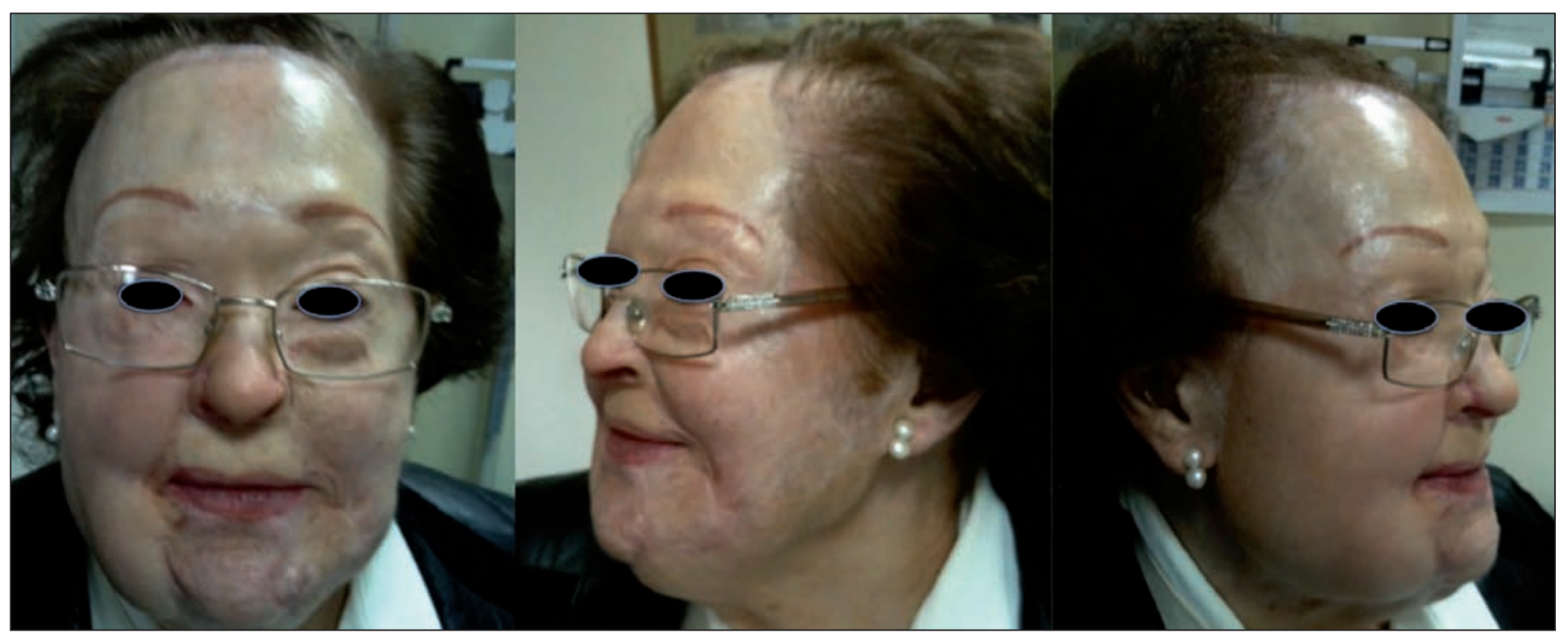

Fig. 9. Reconstrucción de unidades estéticas faciales mediante colgajo radial, pedio e injertos de piel total. Resultado a los 3 meses de la última cirugía (colgajo pedio)

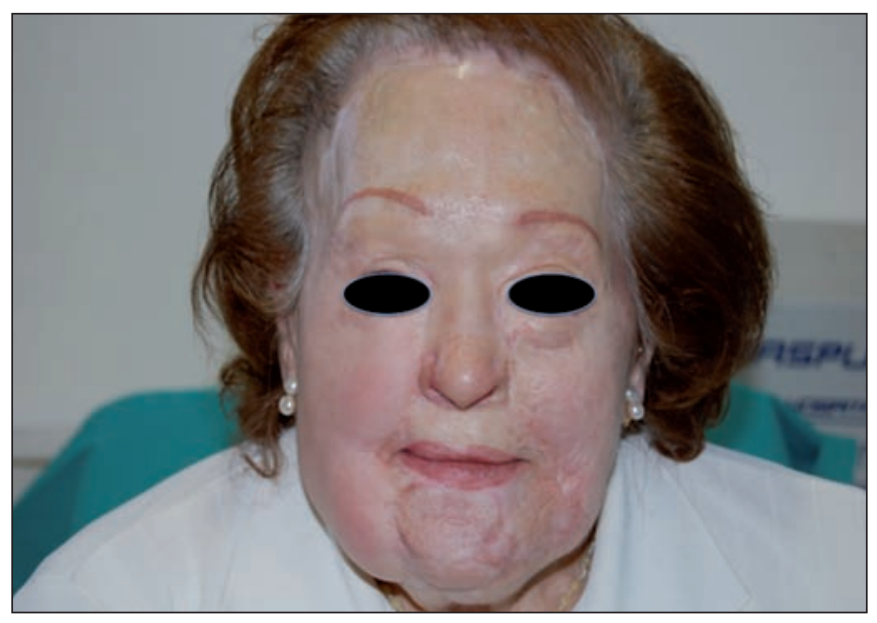

Fig. 10. Resultado de la reconstrucción a los 2 años. Se ha evitado recidiva y progresión de la cicatrización hipertrófica.

\section{Discusión}

Para De Lorenzi y col. (16) la tasa de transferencias de colgajos vascularizados respecto al total de pacientes ingresados en la Unidad de Quemados es del 1,8\%. En nuestra muestra recogida entre agosto de 2001 y agosto de 2013, esta tasa supuso el 1,44\% (total de ingresos de ese periodo de 12 años, 1.458 pacientes, con 21 procedimientos de transferencia de colgajos vascularizados).

Según nuestro estudio, la etiología más frecuente de las quemaduras que precisan reconstrucción con colgajo libre es la eléctrica por alta tensión (en nuestro estudio 8 casos, 38\% del total), lo cual coincide con el estudio de Tarim y col. (12). Esta asociación se debe a la grave afectación de estructuras profundas y exposición de tejidos nobles que producen las quemaduras eléctricas de alta tensión, aunque por lo general la superficie corporal quemada no suele ser muy amplia.

La tasa de complicaciones en nuestra muestra de pacientes quemados fue alta, con una tasa de complicacio- nes del 28,55\%, correspondiendo el 19,05\% a complicaciones mayores y el $9,5 \%$ a complicaciones menores. Es mayor que la tasa de complicaciones en colgajos libres que muestran ciertos estudios previos. El estudio de Pohlenz y col. (17) analiza los resultados de 1.000 colgajos aplicados para reconstrucción de cabeza y cuello, con una tasa de fallo del colgajo total o parcial del 7,6\%, similar a la de 7,2\% (3,9\% la pérdida total y $3,3 \%$ la pérdida parcial) que muestra el estudio de Cornejo y col. sobre las complicaciones de 179 colgajos libres aplicados a múltiples tipos de reconstrucción (18).

La incidencia de complicaciones postquirúrgicas podría variar en las diferentes poblaciones e indicaciones donde se realizan transferencias de colgajos vascularizados según sus características y las comorbilidades de los pacientes. Es así como en casos extremos, tales como poblaciones diabéticas, el índice de complicaciones se puede disparar como muestra el estudio de Lee y col., en el que analizan los resultados de la reconstrucción mediante colgajos libres de un grupo de 33 pacientes diabéticos tipo 2 con lesiones severas de tejidos blandos en extremidades, presentando una tasa de complicaciones mayor del $50 \%$, con un $30,3 \%$ de pérdida total del colgajo y un $24 \%$ de necrosis parcial (19).

En cuanto a la tasa de complicaciones y sus variaciones según el momento de la cirugía, que es el principal objetivo de nuestro estudio, Ofer y col. afirman en su estudio que la tasa de pérdida de colgajo libre es mayor en el periodo primario-temprano (entre 5-21 días tras el traumatismo) (8), afirmación que también está presente en otros estudios como el de Oni y col. (5) o los de Sauerbier y Baumeiter $(4,6)$.

Nuestros resultados son congruentes con los estudios comentados $(4-6,8)$ : mientras que no se realizó ninguna reconstrucción en el periodo primario inmediato en nuestra muestra, los fracasos de colgajos se concentraron en el mismo periodo "de riesgo" que el de los estudios de 
Ofer, Oni, Sauerbier y Baumeiter, ya que en el periodo primario-temprano se situó el $50 \%$ de los fracasos de los colgajos de nuestro estudio (2 pérdidas totales de colgajo), mientras que no se registraron complicaciones mayores en las reconstrucciones realizadas en el periodo primario intermedio (entre el día 21 y la $6^{\mathrm{a}}$ semana). Un $25 \%$ de las necrosis ( 1 caso) se produjeron en reconstrucciones secundarias (más de 6 semanas) y otro $25 \%$ ( 1 caso) en cirugía de secuelas. Al analizar los datos en las tablas de contingencia estudiamos la asociación entre las variables "complicaciones mayores" y el "periodo de reconstrucción", y aplicamos el test de Chi-cuadrado con la simulación de Montecarlo obteniendo un valor de Chicuadrado de 3,5515 con p-valor de 0,3913 . No encontramos asociación estadísticamente significativa, probablemente debido al pequeño tamaño muestral del estudio y al elevado número de categorías de las variables.

Por todo ello, realizar la reconstrucción en el periodo primario temprano (5-21 días tras el traumatismo) podría presentar efectivamente un aumento de riesgo de complicaciones tal y como señalan los estudios de Sauerbier, Oni, Baumeiter y Ofer $(4-5,8)$ y como apoyan nuestros resultados; esto podría ser debido a los elevados niveles de inflamación locales y sistémicos así como a las importantes alteraciones fisiológicas presentes en este periodo en los pacientes quemados, que pondrían en peligro la viabilidad de los colgajos. Fuera de este periodo, la tasa de complicaciones retornaría a un nivel similar a la general. Apoyando esta hipótesis, el estudio de Ofer y col. (8) muestra una incidencia muy baja de complicaciones en el periodo primario inmediato (en los 5 primeros días); sin embargo en nuestra experiencia, es difícil llevar a la práctica una reconstrucción en los primeros 5 días porque con frecuencia la estabilización sistémica del paciente quemado nos lleva más de esos 5 días.

Por el contrario, en su revisión de 38 colgajos libres fasciocutáneos, Pan y col. presentan unas tasas de supervivencia del 100\% independientemente del momento en el que se realizara la reconstrucción de la quemadura, aunque sí registran un 8\% de necrosis parcial de los colgajos. Ellos defienden que si se realiza un correcto desbridamiento y control de las infecciones, así como una correcta técnica microquirúrgica, no hay aumento de riesgo de fallo del colgajo en las reconstrucciones de quemaduras (7).

Históricamente se ha achacado el incremento del fallo de los colgajos microquirúrgicos en los pacientes con quemaduras eléctricas y con quemaduras térmicas importantes, a la necrosis progresiva de los tejidos así como a daños vasculares (daños de la íntima y la capa media, oclusión vascular, formación de aneurismas, arteritis, trombosis, adelgazamiento de vasos, etc.). Sin embargo, estos hechos no deberían ser un inconveniente si realizamos la reconstrucción de un modo racional: tras los desbridamientos sucesivos (ya que muchas veces la lesión no está claramente delimitada al inicio), realizaremos la reconstrucción una vez tengamos claro que el tejido que queda es viable y está en buenas condiciones, con un control estricto de las infecciones (20), y evitando estados inflamatorios y de edema de los tejidos. Por otro lado hemos de seleccionar vascularización claramente viable: hay que asegurar que las suturas vasculares se hacen en regiones inmediatamente adyacentes a la lesión pero que conserven una buena viabilidad. Kuo y col. (21) mostraron en un modelo experimental con conejo que los vasos separados $3 \mathrm{~cm}$ del borde de las quemaduras, presentan características normales en cuanto a elasticidad, características del endotelio, etc.

Otras causas de fallo del colgajo podrían ser la existencia de infecciones, un inadecuado desbridamiento, incompetencia o insuficiencia de la/s perforante/s, o tras el proceso de adelgazamiento del colgajo (en casos en los que éste se realiza en el primer tiempo quirúrgico). Por otro lado, también influyen en el fracaso o en la supervivencia algunos factores fisiopatológicos del propio paciente, como es el caso de la anemia preoperatoria. El estudio de Bradford Hill y col. afirma que hay una asociación directa entre el grado de anemia y el riesgo de pérdida de los colgajos. Este trabajo analiza 156 colgajos libres y relaciona la tasa de pérdida del colgajo con los niveles de anemia preoperatoria, obteniendo una relación directa estadísticamente significativa (22). La anemia de base, presente en muchos pacientes quemados secundariamente a los esfuerzos de resucitación, a la expansión iatrogénica de volumen plasmático y a la reserva fisiológica disminuida del paciente quemado, podría ser un factor determinante en la pérdida de los colgajos. Los efectos de la anemia en la dinámica sanguínea provocarían hemodilución y menor viscosidad, que según la ecuación de Poiseuille y la constante de Reynolds, reducirían el flujo laminar aumentando el flujo turbulento, y con ello la posibilidad de trombosis arteriales.

\section{Conclusiones}

Según nuestro estudio, y coincidiendo con otros estudios previos, la cirugía reconstructiva microquirúrgica del paciente quemado se ha de realizar fuera del periodo primario temprano (entre el día 5 y el 21 tras la quemadura) ya que en este periodo hay una incidencia más alta de complicaciones.

Por otro lado, comparando los resultados y complicaciones de nuestra muestra de pacientes quemados con los datos de resultados y complicaciones de reconstrucciones con colgajos libres presentados en la literatura médica, la tasa de complicaciones en los quemados podría estar aumentada respecto a la tasa de complicaciones en reconstrucciones con colgajo libre en la población general.

A nuestro entender, este estudio es interesante para la comunidad científica en general y específicamente para los cirujanos plásticos especializados en el tratamiento de pacientes quemados, tanto por las cuestiones que plantea como por las nuevas dudas que se generan. Creemos 
que sería interesante realizar un estudio multicéntrico entre las distintas Unidades de Quemados que nos ayudara a comprender mejor la relación entre la fisiopatología de las quemaduras, la tasa de complicaciones en reconstrucciones con colgajos libres y la relación de estos elementos con el momento de la cirugía.

\section{Dirección del autor}

Dra. María Eloisa Villaverde Doménech

C/ Puerto Rico $\mathrm{N}^{\circ} 32,4^{\circ}-8$

46006 Valencia, España

correo electrónico: eloisavillaverde@ outlook.es

\section{Bibliografía}

1. Sharzer, L.A., O'Brien, B.M., Horton, C.E., Adamson, J.E., Mladick, R.A., Carraway, J.H., et al.: Clinical applications of free flap transfer in the burn patient. $J$ Trauma. 1975;15(9):766-771.

2. Harii K, Ohmori K, Ohmori S.: Utilization of free composite tissue transfer by microvascular anastomoses for the repair of burn deformities. Burns. 1975; 1(3): 237244.

3. Abramson, D.L., Pribaz, J.J., Orgill, D.P. : The use of free tissue transfer in burn reconstruction. $J$ Burn Care Rehabil. 1996t;17(5):402-408.

4. Baumeister, S., Köller, M., Dragu, A., Germann, G., Sauerbier, M.: Principles of microvascular reconstruction in burn and electrical burn injuries. Burns. 2005; 31(1):92-98.

5. Oni, G., Saint-Cyr, M., Mojallal, A.: Free tissue transfer in acute burns. J Reconstr Microsurg. 2012; 28 (2); 77-84.

6. Sauerbier M1, Ofer N, Germann G, Baumeister S.: Microvascular reconstruction in burn and electrical burn injuries of the severely traumatized upper extremity. Plast Reconstr Surg. 2007;119(2):605-615.

7. Pan, C.H., Chuang, S.S., Yang, J.Y.: Thirty-eight free fasciocutaneous flap transfers in acute burned-hand injuries. Burns. 2007; 33 230-235.

8. Ofer, N., Baumeister, S., Megerle, K., Germann, G., Sauerbier, M.: Current concepts of microvascular reconstruction for limb salvage in electrical burn injuries. J Plast Reconstr Aesthet Surg. 2007;60(7):724-30. Epub 2007 May 4.

9. Hu, X.H., Quin, F.J., Chen, Z., Shen, Z.Y., Shen, Y.M.: Combined rectus abdominis muscle/paraumbilical flap and lower abdominal flap for the treatment of type III circumferential electrical burns of the wrist. Burns. 2013; 39 1631-1638.
10. Kesiktas, E., Eser, C., Gencel, E., Efe Aslaner, E., Yavuz, M.: Reconstruction of transhumeral amputation stumps with ipsilateral pedicled latissimus dorsi myocutaneous flap in high voltage electrical burns. Burns. 2015; 41 401-407.

11. Aghakhani, K., Heidari, M., Morteza Tabatabaee, S., Abdolkarimi, L.: Effect of current pathway on mortality and morbidity in electrical burn patients. Burns. $2015 ; 172-176$.

12. Tarim, A., Ezer, A.: Electrical burn is still a major risk factor for amputations. Burns. 2013; 39 354-357.

13. Sarikaya A1, Aygit AC.: Combined $99 \mathrm{mTc}$ MDP bone SPECT and 99mTc sestamibi muscle SPECT for assessment of bone regrowth and free muscle flap viability in an electrical burn of scalp. Burns. 2003;29(4):385-388.

14. Fried, M., Rosenberg, B., Tuchman, I., Ben-Hur, N., Yardeni, P., Sternberg, N., et al.: Electrical burn injury of the scalp - bone regrowth following application of latissimus dorsi free flap to the area. Burns. 1991; 17(4): 338-339.

15. Hofer, S., Mureau, M.: Improving Outcomes in Aesthetic Facial Reconstruction. Clin Plast Surg. 2009;36(3): 345-354

16. De Lorenzi, F., Van der Hulst, R., Boeckx, W.: Free flaps in burn reconstruction. Burns. 2001;27(6):603-612.

17. Pohlenz, P., Klatt, J., Schön, G., Blessmann, M., Li, L., Schmelzle, R.: Microvascular free flaps in head and neck surgery: complications and outcome of 1000 flaps. Int J Oral Maxillofac Surg. 2012;41(6):739-743.

18. Cornejo, A.,Ivatury, S., Crane, C.N.,Myers, J.G., Wang, H.T.: Analysis of Free Flap Complications and Utilization of Intensive Care Unit Monitoring. J Reconstr Microsurg 2013;29:473-480.

19. Lee, Y.K.,Park, K.Y., Koo, Y.T., Baek, R.M., Heo, C.Y., Eun, S.C., Lee, T.S., Lee, K.M., Kim, B.K.: Analysis of multiple risk factors affecting the result of free flap transfer for necrotising soft tissue defects of the lower extremities in patients with type 2 diabetes mellitus. J Plast Reconstr Aesthet Surg. 2014;67(5):624-628.

20. Saracoglu, A. Et Al.: Prognostic factors in electrical burns: A review of 101 patients. Burns. 2014; 40702 707.

21. Kuo et Al.: Experimental study of free flap transplantation after debridement in early stage of electric burn. Zhonghua Zheng Xing Shao Shang Wai Ke Za Zhi 1990; 318: 285-287.

22. Bradford Hill, J. Et Al.: Preoperative Anemia Predicts Thrombosis and Free Flap Failure in Microvascular Reconstruction. Ann Plast Surg, 2012; 69 (4); 364-367. 